\title{
ON THE GROWTH OF AMMONIUM NITRATE(III) CRYSTALS
}

\author{
L.J.P. VOGELS, H.A.M. MARSMAN, M.A. VERHEIJEN and P. BENNEMA \\ RIM Laboratory of Solid State Chemistry, University of Nijmegen, Toernooiveld, 6525 ED Nijmegen, The Netherlands
}

and

\section{ELWENSPOEK}

Faculty of Electrotechnics, Technical University of Twente, P.O. Box 217, 7500 AE Enschede, The Netherlands

Received 9 September 1989; manuscript received in final form 29 November 1989

The growth rate of $\mathrm{NH}_{4} \mathrm{NO}_{3}$ phase III crystals is measured and interpreted using two models. The first is a standard crystal growth model based on a spiral growth mechanism, the second outlines the concept of kinetical roughening. As the crystal becomes rough a critical supersaturation can be determined and from this the step free energy. The step free energy versus temperature turns out to be well represented by a Kosterlitz-Thouless type model. Further a phenomenological treatment of some peculiar growth observations is given.

\section{Introduction}

Ammonium nitrate has been an object of investigation for over a century because of its use as a fertilizer and explosive [1-5]. At atmospheric pressure $\mathrm{NH}_{4} \mathrm{NO}_{3}$ is a polymorphic compound. In the following scheme the temperature intervals of each phase are given [6]:

$$
\begin{aligned}
& \mathrm{I}-\left(125.2^{\circ} \mathrm{C}\right)-\mathrm{II}-\left(84.2^{\circ} \mathrm{C}\right)-\mathrm{III}-\left(32.3^{\circ} \mathrm{C}\right)-\mathrm{IV}- \\
& \left(-17.5^{\circ} \mathrm{C}\right)-\mathrm{V} .
\end{aligned}
$$

Especially the phase transitions and recrystallization give rise to many problems, all of which are important to control when growing $\mathrm{NH}_{4} \mathrm{NO}_{3}$ crystals from the bulk [7-9]. In this matter not only growth mechanisms but also the morphology of ammonium nitrate is believed to contain important information for the understanding of growth. Recently, studies have been carried out using statistical mechanical and thermodynamical theories to predict the morphology of crystals $[10,11]$.

As long as the temperature is below a critical roughening transition temperature, a crystal face grows as a "flat" face [12]. The standard growth of crystals on crystal faces $(h k l)$ takes place by a layer by layer growth mechanism like two dimensional nucleation or spiral growth. However as the supersaturation $\sigma$ becomes larger than a critical $\sigma_{c}$ crystals grow with an additional growth mechanism. Now the crystal surface becomes kinetically rough and the incorporation of growth units is dominated by volume and/or surface diffusion. This effect is called kinetical roughening and causes crystal faces to become rounded [13-16].

In section 2 of this paper the theory of kinetical roughening will be discussed as well as an outline of the spiral growth models. Also the relation between kinetical roughening at the one hand and crystal structure and morphology at the other hand will be demonstrated. Section 3 will show the experimental set-up as well as the experimental techniques used. Section 4 will give results of the experiments and further a phenomenological treatment of the experiments. Section 5 will contain a comparison between theory and experiment and some concluding remarks. 


\section{Theory}

$\mathrm{NH}_{4} \mathrm{NO}_{3}$ (III) has the orthorhombic space group $\mathrm{Pbnm}$ containing four formula units per unit cell. Carrying out a so-called periodic bond chain (PBC) analysis only first nearest neighbour bonds were taken into account. The PBC analysis of $\mathrm{NH}_{4} \mathrm{NO}_{3}$ (III) showed that one could construct the following $F$ (flat) faces:

$\{111\},\{220\},\{002\},\{101\},\{200\}$ and $\{011\}$.

These faces were also experimentally found [11].

The growth habit of most $\mathrm{NH}_{4} \mathrm{NO}_{3}$ (III) crystals is determined by large $\{101\}$ prismatic faces and by small $\{111\}$ pyramidal faces. Considering energies this implies a higher surface energy for the $\{111\}$ faces as compared to the $\{101\}$ faces and hence a smaller step free energy for the $\{111\}$ faces as compared to the $\{101\}$ faces. The step free energy plays an important role in the concept of kinetical roughening.

Since Frank postulated in 1949 that crystals grow by a spiral mechanism and the subsequent development of the BCF theory $[17,18]$, many experiments have been carried out to test these theories. It was found that at larger supersaturation crystals could also grow by a two-dimensional nucleation mechanism. To reveal both mechanisms several investigations have been done [19-21].

Thermodynamically, the relative supersaturation $\sigma$ is given as:

$\sigma=\Delta \mu / k T$

with $\Delta \mu$ the difference in chemical potential per growth unit of solute molecules in the crystal and mother phase, $k$ the Boltzmann constant and $T$ the absolute temperature. The relative supersaturation is further given by:

$\sigma \cong \frac{\Delta T}{T_{\mathrm{eq}}^{2}} \frac{\Delta h}{R} \quad$ or $\quad \sigma=-1907 \frac{\Delta T}{T_{\mathrm{eq}}^{2}}$,

with $T_{\text {eq }}$ the equilibrium temperature $(\mathrm{K})$ of the system, $\Delta T$ the difference between $T_{\text {eq }}$ and $T_{\text {growth }}$, $R$ the gas constant and $\Delta h$ the latent heat of fusion. The density of steps on the surface will be proportional to the supersaturation $\sigma$ and to the growth velocity $R$ perpendicular to a crystal face $(h k l)$, which can be described as a function of the supersaturation $\sigma$. This is the so-called BCF relation between $R$ and $\sigma[17,22]$ :

$R \simeq \sigma^{2} \tanh \left(\sigma_{1} / \sigma\right)$

where $\sigma_{1}$ is a constant. The mechanism before could be referred to as a standard crystal growth mechanism.

Recent studies [23-25] showed that impurities have a dramatic influence on the growth $(\mathrm{KH}$ phthalate) and morphology $\left(\mathrm{KH}_{2} \mathrm{PO}_{4}\right)$ of crystals. Spirals and steps get blocked or change in habit and mainly in case of a small supersaturation the growth velocity $R$ of a face $(h k l)$ becomes 0 . It then is possible that the morphology changes; e.g., prismatic faces get blocked, while pyramidal faces still grow $\left(\mathrm{KH}_{2} \mathrm{PO}_{4}\right)$. We here encounter a second growth habit.

The free energy per growth unit of a step at a crystal face, $k T \gamma$, plays an important role in crystal growth. $k T \gamma$ may be seen as the physical quantity characterizing the physical state of the surface. At a temperature $T_{\mathrm{r}}$, a roughening transition for a crystal face $(h k l)$ takes place, $\gamma$ becomes 0 and the crystal is microscopically rough. As $\gamma=0$, the nucleation barrier vanishes and it becomes possible for the growth units to incorporate directly on the crystal surface and thermal fluctuations create a great number of steps on the crystal surface. Above $T_{\mathrm{r}}$ crystal faces become macroscopically rounded. Below the roughening temperature $T_{\mathrm{r}}$, where $\gamma>0$, only two-dimensional nucleation and/or spiral growth is possible, the crystal is flat and grows by a layer-by-layer mechanism. $T_{\mathrm{r}}$ is a property of the different crystal faces and thus certain faces on a crystal are rough at a given temperature and others are flat. Rough faces do not appear on the crystal form, while flat faces possibly appear. A well-known parameter used to characterize $T_{\mathrm{r}}$ is the $\alpha$-factor of Jackson [26-29], where $\alpha_{j}$ is the potential energy in a slice with orientation $(h k l)$ divided by the temperature.

On the subject of $\gamma$, computer simulations were carried out which supported the idea of a Koster- 
litz-Thouless [30] type phase transition of $\gamma$. As $T$ approaches $T_{\mathrm{r}}$, a relation for $\gamma$ is given by [31]:

$\gamma \simeq \exp \left[-c\left[\frac{T_{\mathrm{r}}}{T_{\mathrm{r}}-T}\right]^{1 / 2}\right]$.

Besides the above-mentioned phenomena, organic crystals can also become macroscopically rough below $T_{\mathrm{r}}$, as the supersaturation becomes larger than a certain value, $\sigma_{c}[13,14,32,33]$. Microscopically, crystals remain flat. Normally there is a flux of growth particles to and from the crystal surface. It then is possible that at a certain chemical potential difference $\Delta \mu$, or at a certain critical supersaturation $\sigma_{c}$, the flux towards the surface becomes dominant. Then the growth will be dominated by an incoming flux. This phenomenon occurs if the step free energy on a crystal face is small enough, so that the before mentioned nucleation barrier which controls the growth of flat crystal faces can be overwhelmed by a chemical potential difference $\Delta \mu$. A direct incorporation of growth units is then possible. It has been found that above a critical $\sigma$, the crystal face becomes rounded and the growth rate is described by:

$R=R_{0}\left(\sigma-\sigma_{\mathrm{c}}\right), \quad \sigma>\sigma_{\mathrm{c}}$.

A similar relation is found by Monte Carlo simulations [34].

In a recent survey on kinetical roughening, also a useful relation for isotropic growth units between $\sigma_{\mathfrak{c}}$ and $\gamma$ is derived $[13,14]$ :

$\sigma_{\mathrm{c}}=\pi(\gamma)^{2}$.

$\sigma_{\mathrm{c}}$ can easily be determined by measuring the growth velocity $R$ versus $\sigma$ and extrapolating the linear part of the curve to $R=0$ or by observing changes in the growth form.

\section{Experiments}

Ammonium nitrate solutions used in our experiments were prepared with Merck P.A. $\mathrm{NH}_{4} \mathrm{NO}_{3}$ and demineralized water. Dust particles were prevented to enter the observation cell by

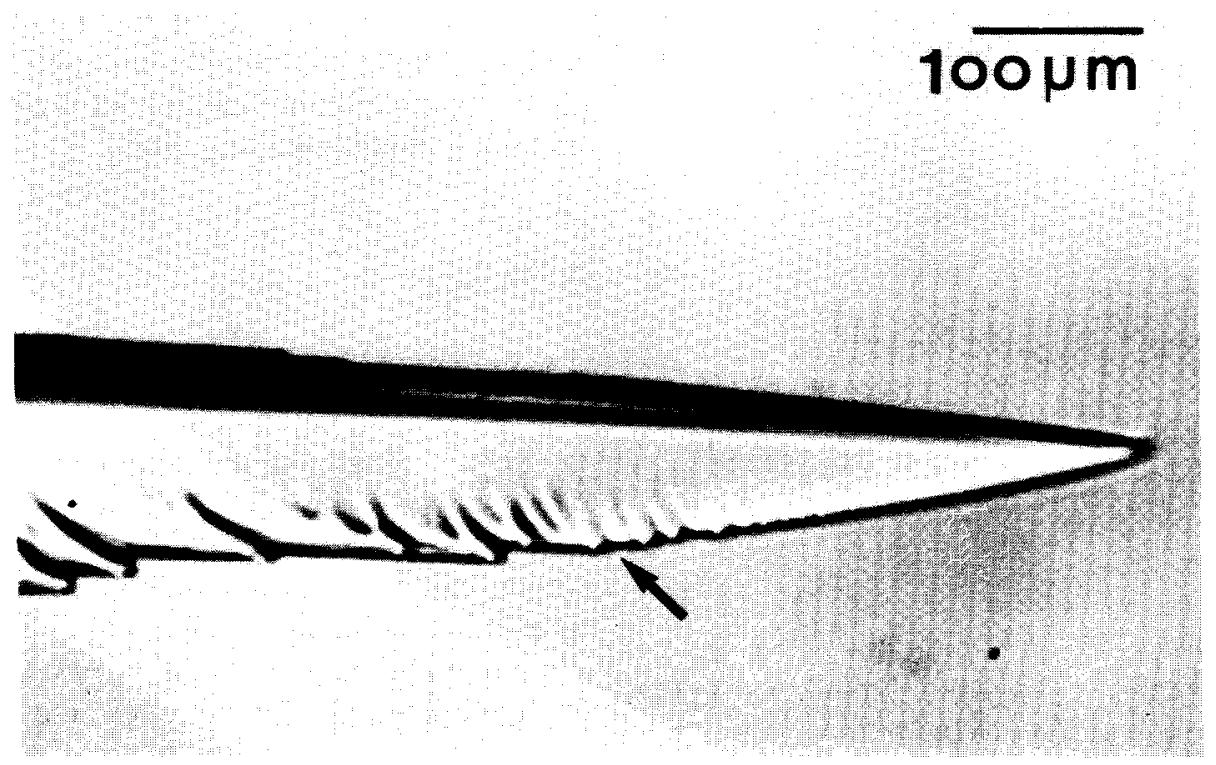

Fig. 1. "Scalpel"-growth of $\mathrm{NH}_{4} \mathrm{NO}_{3}$ (III). The $\{111\}$ face is kinetically rough, it becomes rounded. The crystal forms facets again as the local supersaturation becomes smaller (indicated by the arrow) 
pouring the solution through a millipore $(d=0.22$ $\mu \mathrm{m})$ filter. The observations of growth velocities and growth forms were done in double-walled thermostated glass cells of cylindric form $(r \approx 60$ $\mathrm{mm}$, height $\approx 5 \mathrm{~mm}$ ) using polarization microscopy. Thus it was possible to control the temperature within $\pm 0.01^{\circ} \mathrm{C}$. All experiments were performed in stagnant solutions and the supersaturation was increased to a maximum of $5 \%$. Because of heat diffusion problems experiments were carried out using a small temperature gradient of about $0.05^{\circ} \mathrm{C} / \mathrm{min}$ and only after waiting for several minutes to be sure that the measured temperature of the thermostate was the same as that of the inside of the observation cell, the equilibrium temperature of the system.

Crystals used for observation were obtained by nucleating crystals, of phase IV or phase III, and subsequently dissolving all but one crystal. To stabilize the solution and to get rid of concentration gradients one had to wait a while. It is certain that only phase III crystals could remain in the solution because relatively small crystals of phase IV have a lifetime of a few minutes in phase III solutions before recrystallization takes place. Growth forms typically had $\{101\}$ prismatic and \{111\} pyramidal faces [11].

As the crystal became kinetically rough the pyramidal faces became rounded and typical "scalpel"-like crystals were observed (fig. 1) $[2,4]$.

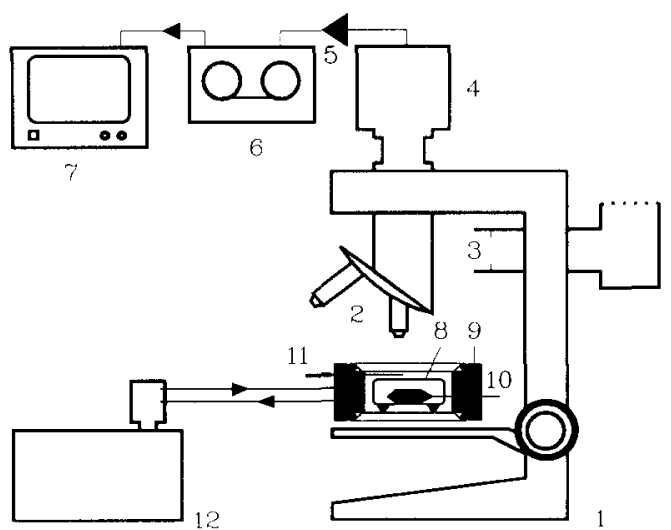

Fig. 2. Experimental set-up: (1) microscope; (2) objective; (3) aperture diafragm; (4) video camera; (5) contrast enhancer; (6) video recorder; (7) monitor; (8) growth cell; (9) vessel; (10) crystal; (11) Pt-100 resistor; (12) thermostat.

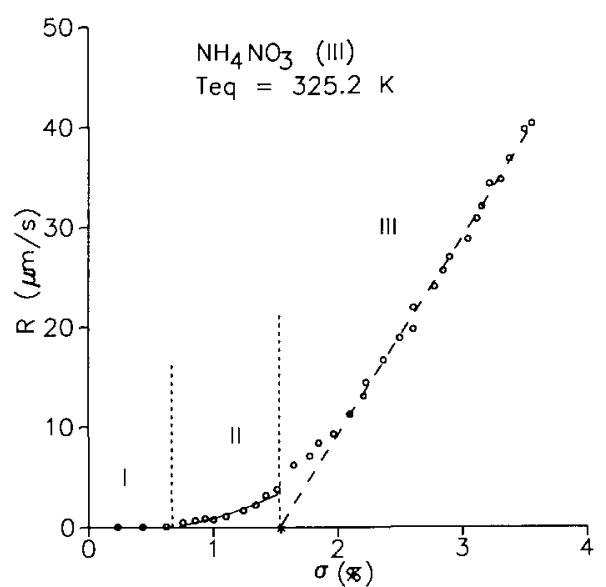

Fig. 3. Growth velocity $R$ of $\{111\}$ faces versus supersaturation $\sigma$. Three distinct growth regions can be seen. In region I there is no or almost no growth and in region II the crystal grows by a spiral or stepped growth mechanism. From region III the critical supersaturation can be determined as $\sigma_{c}=1.5 \%$.

These crystals were used to measure growth velocities. Also some experiments with intentional impurities were carried out. A recording of growth forms and velocities was made on video tape using a videocamera (fig. 2). Afterwards growth velocities and forms could be determined on a monitor.

Because the volume of the remaining crystal was very small compared to the volume of the cell, the changes in concentration in the liquid during the experiment were negligible.

\section{Results}

\subsection{Growth measurements}

First of all growth measurements of $R$ versus $\sigma$ were carried out. We observed the $\{111\}$ pyramidal face. Fig. 3 shows a typical growth curve which can be divided into three areas. In area I the growth velocity equals zero $(\sigma<0.2 \%)$ or is very small $(0.2 \%<\sigma<0.7 \%)$, probably due to impurities blocking the steps on the surface [35]. Also the $\{111\}$ face has formed no scalpel yet. Area II is the area where crystals grow by a spiral mechanism. A fit of eq. (3) with this part of the 
curve gives a good correlation. As a guide to the eye the fit of

$R=R_{0}+k \sigma^{2} \tanh \left(\sigma_{1} / \sigma\right)$

with $\sigma_{1}=18 \%, \quad R_{0}=-0.9 \mu \mathrm{m} / \mathrm{s}$ and $k=$ $1.9 \mu \mathrm{m} / \mathrm{s}$ is drawn. $R_{0}$ is probably related to area I, where the growth velocity equals zero. Area II can be seen as an intermediate growth region, as $\sigma<\sigma_{\mathrm{c}}$ only spiral growth is present, while for $\sigma>\sigma_{\mathrm{c}}$ (area III) also a diffusion growth mechanism is present. In area II, scalpels are being formed and at $\sigma=\sigma_{c}$ kinetical roughening occurs. In area III, the crystal faces are rounded (fig. 1). Here the growth of the $\{111\}$ faces is dominated by diffusion processes. From area III, we are able to determine $\sigma_{\mathrm{c}}$ by extrapolating the linear part of the curve to $R=0\left(\sigma_{\mathrm{c}}=1.5 \%\right)$, which is in good agreement with $\sigma_{\mathrm{c}}$ determined by the observed roughening of the scalpel-like growth form.

\subsection{Temperature dependence of the critical super- saturation $\sigma_{c}$}

The above described experiment has been carried out several times with different vessels. All of these vessels had a different $T_{\text {eq }}$. Thus we were able to determine a temperature dependence of $\sigma_{c}$,

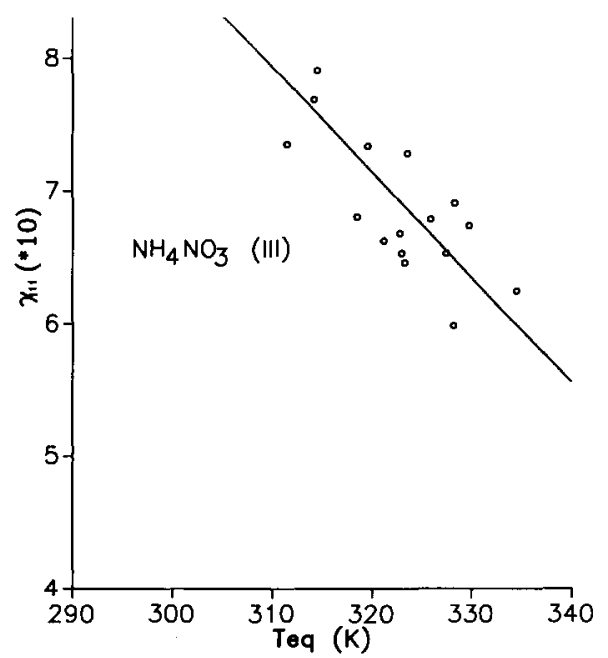

Fig. 4. Step free energy per growth unit divided by $k T\left(\gamma_{111}\right)$ versus equilibrium temperature $T_{\text {eq }}$ of the different solutions. An estimate for $T_{\mathrm{r}}$ is $440 \pm 20 \mathrm{~K}$. and herewith of $\gamma_{111}$, as can be seen in fig. 4 . Results of solutions with intentional impurities fit well in the curve of fig. 4 . As the growth process in area III is probably diffusion controlled impurities would have no effect on the growth velocity, assuming the influence of the step free energy is negligible. For areas I and II, $R$ is smaller compared to the same solutions without intentional impurities.

Comparing the experimental results with the relation for $\gamma$ given in eq. (4) the agreement is satisfying. Note however that $T \ll T_{\mathrm{r}}$. The full curve represents

$\gamma=110 \exp \left[-c\left(\frac{T_{\mathrm{r}}}{T_{\mathrm{r}}-T}\right)^{1 / 2}\right]$

with $T_{\mathrm{r}}=440 \pm 20 \mathrm{~K}$ and $c=1.2 \pm 0.2$ and, as can be seen, the theoretical data fit the experimental data very well. It should be noted that $T_{\mathrm{r}}$ has no physical meaning as $\mathrm{NH}_{4} \mathrm{NO}_{3}$ (III) is not stable at this temperature.

Note that the temperature range investigated is rather small, so that the agreement of $\gamma$ as a function of $T$ with the form in eq. (4) might be fortuitous. We note that the $\{111\}$ face of $\mathrm{NH}_{4} \mathrm{NO}_{3}$ (III) behaves similar to metallic systems where $\gamma(T)$ is similar [36], but differs from organic systems, where $\delta \gamma / \delta T \cong 0$ [13].

\subsection{Width (r) dependence of the growth velocity}

The growth velocity $R$ is not only a function of $\sigma$. Under equal conditions very different results were obtained. A possible explanation is given below.

Since the habit of $\mathrm{NH}_{4} \mathrm{NO}_{3}$ could differ under equal conditions, it was obvious that the form of the crystal could be a parameter influencing $R$. As a measure of difference the width $r$ of the crystal at a fixed distance of $-300 \mu \mathrm{m}$ from the top is taken. In fig. 5 the growth velocity versus the width is given. As $\sigma>\sigma_{\mathrm{c}}$ ( $\sigma_{\mathrm{c}}$ being $\left.1.5 \%\right), R$ increases with increasing $r$ and for $\sigma<\sigma_{\mathrm{c}}, R$ is independent of $r$.

As $\sigma<\sigma_{\mathrm{c}}$ there are no or small differences in growth velocity as can be expected. Crystal growth is determined by spiral or stepped growth and the 


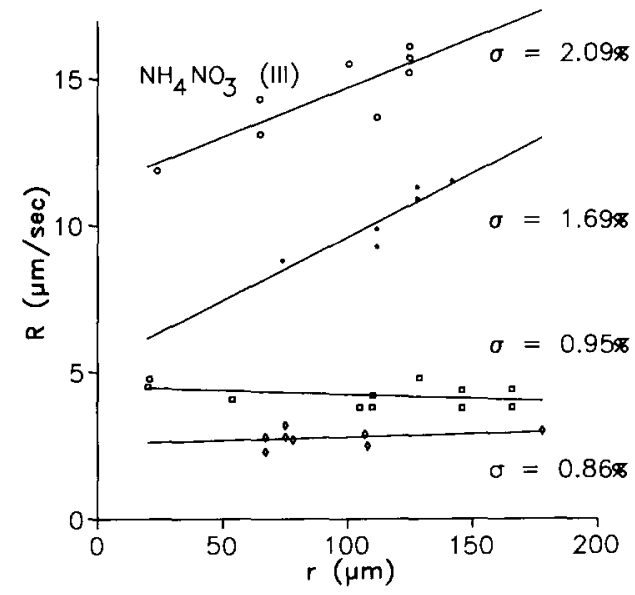

Fig. 5. Growth velocity $R$ of $\{111\}$ faces of $\mathrm{NH}_{4} \mathrm{NO}_{3}$ (III) versus width $\mathrm{r}$ at different supersaturations $\sigma . R$ increases with respect to $r$ as $\sigma>\sigma_{\mathrm{c}}$.

density of these should not differ to much as a function of surface area. For $\sigma>\sigma_{\mathrm{c}}$ the results are in contradiction with expectations. If the process would be volume diffusion determined, crystals with small volumes would grow faster than those with a large volume. The influence of diffusion can be characterized by a diffusion length $D_{1}$. The diffusion length $D_{1}$ is given by the form $D_{1}=D_{\mathrm{c}} / R$ with $D_{\mathrm{c}}$ a diffusion constant and $R$ the growth velocity of a crystal face. For $D_{\mathrm{c}}$, a typical value is $10^{-9} \mathrm{~m}^{2} / \mathrm{s}$ [37] and for $R$ growth velocities of $\sim 10 \mu \mathrm{m} / \mathrm{s}$ are found, so $D_{1} \sim 100 \mu \mathrm{m}$, which is of the same magnitude as the width $r$ of a scalpel. Thus if the diffusion mechanism would control growth, scalpels with widths of the order of $D_{1}$ would have comparable growth velocities. Though, if the thickness of a scalpel decreases with its width, it would still be possible to explain the observed growth velocities. Although we were not able to determine the thickness of the scalpels, our estimate is that there is not an essential difference between slim and broad scalpels. So the opposite of what is expected has been found and up till now no satisfactory explanation could be given. We emphasize that although $R$ was dependent on the crystal form, the critical $\sigma_{\mathrm{c}}$ determined from different scalpels growing in the same vessel with the same $T_{\text {eq }}$ was always the same.

\subsection{Crystals during growth}

As the scalpels are formed the width of the scalpel gradually changed $\left(\sigma>\sigma_{\mathrm{c}}, \sigma_{\mathrm{c}}=1.5 \%\right), r$ seems to be a linear function of $\sigma$. This might be due to the fact that crystals grow faster at a higher $\sigma$. Fig. 6 shows a $T$ dependence of the width of the scalpels. As $\sigma<\sigma_{\mathrm{c}}$ the width is almost constant. At constant $T_{\text {growth }}(\sigma \simeq 1.8 \%)$ the width of the scalpel did not change noticeably for over fifteen minutes.

\subsection{Observations}

Many interesting growth observations occurring on the scalpel faces were made. Some of these will be discussed here.

After studying numerous scalpels, a satisfactory explanation about the formation could be given. It is presumed that instabilities on the crystal at sites where the relative supersaturation or diffusion gradient is large cause scalpels to emerge. Thus the top and the base corners of the pyramid are the most favourable positions to emerge scalpels from. It could not be explained why from the top of the pyramid no scalpels emerge; the top area might be too small. No macrosteps moving towards the $\{111\}$ edge of the $\{101\}$ surfaces were observed before a scalpel emerged, so it is not likely that overhanging layers are responsible for

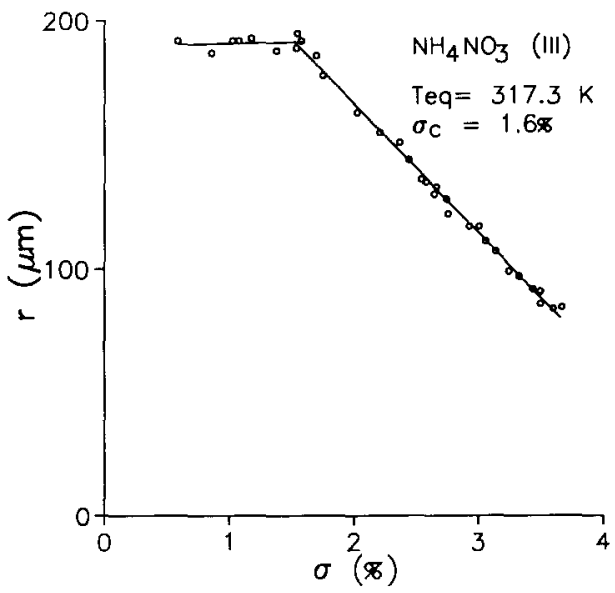

Fig. 6. Width $(r)$ of a scalpel versus supersaturation $\sigma$. As $\sigma>\sigma_{\mathrm{c}}$, the width decreases with $\sigma$ due to diffusion gradients. 


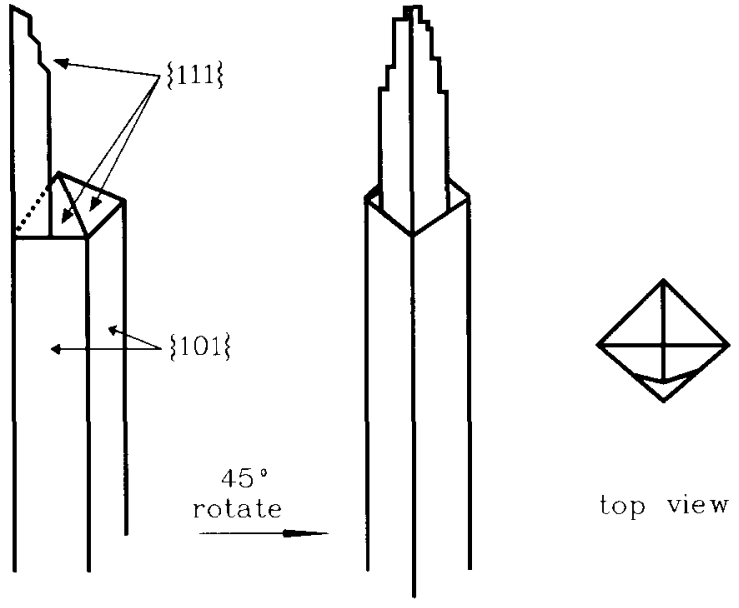

Fig. 7. Schematic view of scalpels. Scalpels emerge from the basic corners of the pyramidal faces.

the protrusion of scalpels. In fig. 7 a schematic view of a scalpel is given.

If we now take a closer look at the top of the scalpel, some effects can be seen. First, at a certain distance from the top, the scalpel becomes facetted again (fig. 1). Due to diffusion or concentration gradient effects, the local supersatura- tion becomes smaller than the critical supersaturation correlated to kinetical roughening, so that facetting occurs again. The orientations of the facetted part are again $\{111\}$ and $\{101\}$.

On the scalpel faces macrosteps and occasionally spirals were observed (fig. 8). The side faces of a scalpel are $\{101\}$ faces.

\section{Discussion}

From growth experiments it is clear that the $\{111\}$ pyramidal faces of $\mathrm{NH}_{4} \mathrm{NO}_{3}$ (III) have the highest growth velocity. All other faces grow slowly or hardly grow at all. The growth of $\{111\}$ faces takes place in three relatively well-defined areas. Area I shows no growth caused by a blocking of steps and spirals due to non- or intentional impurities. Area II is an intermediate growth region with spiral growth. Area III is the part where crystal faces are kinetically rough. A linear growth region is observed as has also been reported in Monte Carlo simulations [34]. A similar experiment described on a different background has been carried out by Kimura [38].

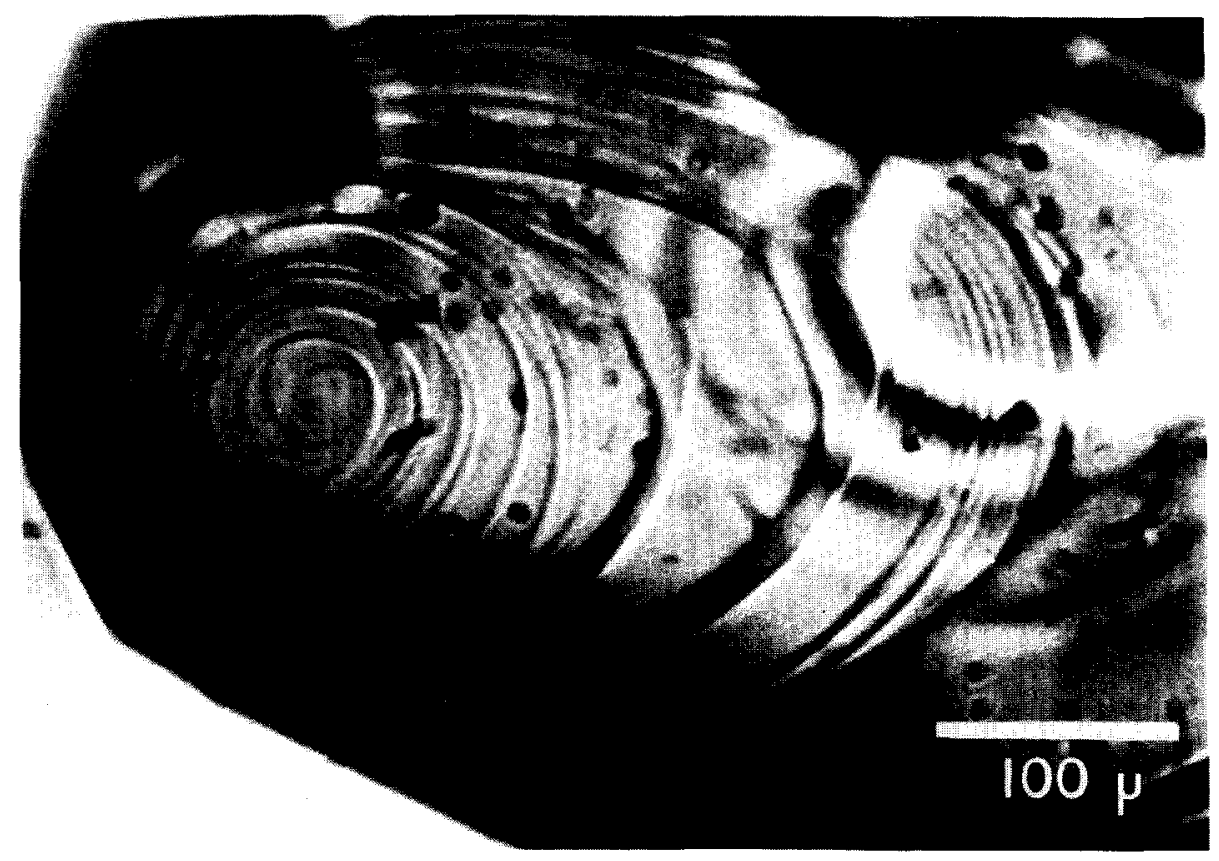

Fig. 8. Spirals on the $\{101\}$ face of $\mathrm{NH}_{4} \mathrm{NO}_{3}$ (III). 
The kinetically rough growth region has been the subject of further investigation. By determining the critical supersaturation $\sigma_{\mathrm{c}}$ at which $\{111\}$ faces become kinetically rough, $\gamma$, the step free energy divided by $k T$, could be determined. Using different solutions with varying equilibrium temperature $T_{\text {eq }}, \gamma_{111}$ could be measured as a function of the absolute temperature. The experimental data found fit the theoretical data derived for a Kosterlitz-Thouless type phase transition surprisingly well.

An observed phenomenon which is not well understood is the fact that slimmer crystals, more or less of the same thickness, grow slower than broader ones, for a supersaturation $\sigma>\sigma_{\mathrm{c}}$. This cannot be explained by a diffusion mechanism. As the supersaturation is smaller than $\sigma_{c}$, growth velocities do not differ.

As the supersaturation rises, the "scalpels" become slimmer, as can be explained by a diffusion gradient model and inherent increase in growth velocity.

Interesting observations have been made on growth forms, changes in form of the "scalpel" and emerging "scalpels". Further investigations have to be done to obtain a deeper understanding of these phenomena.

\section{Acknowledgements}

The authors acknowledge the financial support of the Netherlands Organization for Scientific Research as well as of DSM Netherlands, and Dr. J.P. van der Eerden for stimulating discussions.

\section{References}

[1] Y.N. Tsuchiya and S. Yamamoto, J. Ind. Explosives Soc. Japan 22 (1961) 20.

[2] Y.N. Tsuchiya, J. Ind. Explosives Soc. Japan 23 (1961) 138.

[3] Y.N. Tsuchiya, J. Ind. Explosives Soc. Japan 23 (1962) 78.

[4] J. Whetstone, J. Ind. Chem. 44 (1952) 2663.

[5] L. Phoenix, Brit. Chem. Eng. 11 (1966) 34.

[6] S.B. Hendricks, E. Posnjak and F.C. Kracek, J. Am. Chem. Soc. 54 (1932) 2766.

[7] T. Seiyama and N. Yamazoe, J. Crystal Growth 2 (1968) 255.
[8] C.S. Choi and H.J. Prask, J. Appl. Cryst. 13 (1980) 403.

[9] R.J. Davey, P.D. Guy and A.J. Ruddick, J. Colloid Interface Sci. 108 (1985) 189.

[10] M.H.J. Hottenhuis, L.J.P. Vogels, P. Bennema and M.C. Elwenspoek, J. Crystal Growth, submitted.

[11] L.J.P. Vogels, M.H.J. Hottenhuis, P. Bennema and M.C. Elwenspoek, to be published.

[12] J.P. van der Eerden and H. Knops, Phys. Letters 66A (1978) 334.

[13] M. Elwenspoek and J.P. van der Eerden, J. Phys. A (Math. Gen.) 20 (1987) 669.

[14] M. Elwenspoek, Appl. Phys. A41 (1986) 1.

[15] M. Elwenspoek, P. Bennema and J.P. van der Eerden, J. Crystal Growth 83 (1987) 297.

[16] L.A.M.J. Jetten, Thesis, University of Nijmegen (1983).

[17] W.K. Burton, N. Cabrera and F.C. Frank, Phil. Trans. Royal Soc. London A243 (1951) 299.

[18] F.C. Frank, Disc. Faraday Soc. 5 (1949) 67.

[19] G.J. Abbaschian and M. Eslamoo, J. Crystal Growth 28 (1975) 372

[20] B. Simon, A. Grassi and R. Boistelle, J. Crystal Growth 26 (1974) 77.

[21] S.D. Peteves and G.J. Abbaschian, J. Crystal Growth 79 (1986) 775

[22] P. Bennema and G.H. Gilmer, in: Crystal Growth, An Introduction, Ed. P. Hartman (North-Holland, Amsterdam, 1973) p. 263.

[23] B. Dam, E. Polman and W.J.P. van Enckevort, in: Industrial Crystallization '84, Eds. S.J. Jančić and E.J. de Jong (Elsevier, Amsterdam, 1984) p. 97.

[24] J.W. Noor and B. Dam, J. Crystal Growth 76 (1986) 243.

[25] M.H.J. Hottenhuis and C.B. Lucasius, J. Crystal Growth 78 (1986) 379.

[26] K.A. Jackson, in: Crystal Growth, Ed. H.S. Peiser (Pergamon, Oxford, 1967) p. 17.

[27] R.D. Groot, M. Elwenspoek and P. Bennema, J. Crystal Growth 79 (1986) 817.

[28] M. Elwenspoek, J. Crystal Growth 78 (1986) 353.

[29] J.P. van der Eerden, C. van Leeuwen, P. Bennema, W.L. van de Kruk and B.P. Veltman, J. Appl. Phys. 48 (1977) 2124.

[30] J.M. Kosterlitz and P.J. Thouless, J. Phys. C (Solid State Phys.) 6 (1973) 1181.

[31] J.P. van der Eerden and H. Knops, Phys. Letters 66A (1978) 334 .

[32] L.A.M.J. Jetten, H.J. Human, P. Bennema and J.P. van der Eerden, J. Crystal Growth 68 (1984) 503.

[33] H.J. Human, J.P. van der Eerden, L.A.M.J. Jetten and J.G.M. Odekerken, J. Crystal Growth 51 (1981) 589.

[34] C.K. Ong, A.K. Tan and H.S. Tan, J. Phys. C (Solid State Phys.) 21 (1988) 3655.

[35] N. Cabrera and D.A. Vermilyea, in: Growth and Perfection of Crystals, Eds. R.H. Doremus, B.W. Roberts and D. Turnbull (Wiley, New York, 1958) p. 393.

[36] A. Passerone and N. Eustathopoulos, Acta Met. 30 (1982) 1349.

[37] B. van der Hoek, PhD Thesis, Nijmegen (1983).

[38] H. Kimura, J. Crystal Growth 73 (1985) 53. 